\title{
Oscillation tests for first-order linear differential equations with non-monotone delays
}

Emad R. Attia ${ }^{1,2^{*}}$ (D)

"Correspondence:
dr_emadr@yahoo.com;
er.attia@psau.edu.sa;
emadr@du.edu.eg
'Department of Mathematics,
College of Sciences and Humanities,
Prince Sattam Bin Abdulaziz
University, Alkharj, 11942, Saudi
Arabia
2Department of Mathematics,
Faculty of Science, Damietta
University, New Damietta, 34517,
Egypt

\section{${ }^{*}$ Correspondence:}

1 Department of Mathematics,

College of Sciences and Humanities,

ince Sattam Bin Abdulaziz

Arabia

2Department of Mathematics, University , New Damietta, 34517 ,

\begin{abstract}
We study the oscillation of a first-order linear delay differential equation. A new technique is developed and used to obtain new oscillatory criteria for differential equation with non-monotone delay. Some of these results can improve many previous works. An example is introduced to illustrate the effectiveness and applicability of our results.
\end{abstract}

Keywords: Non-monotone delays; Oscillation; Delay differential equations

\section{Introduction}

Consider the first-order linear delay differential equation

$$
x^{\prime}(t)+p(t) x(\tau(t))=0, \quad t \geq t_{0},
$$

where $p, \tau \in C\left(\left[t_{0}, \infty\right),[0, \infty)\right), \tau(t) \leq t$, such that $\lim _{t \rightarrow \infty} \tau(t)=\infty$.

By a solution of Eq. (1) we mean a continuous function $x(t)$ on $\left[t_{*}, t_{0}\right], t_{*}=\inf _{t \geq t_{0}} \tau(t)$, continuously differentiable on $\left[t_{0}, \infty\right)$, which satisfies Eq. (1) for all $t \in\left(t_{0}, \infty\right)$. As is customary, any solution $x(t)$ of Eq. (1) is called oscillatory if it has arbitrarily large zeros; otherwise it is called non-oscillatory. Equation (1) is said to be oscillatory if all its solutions are oscillatory; otherwise it is called non-oscillatory.

Throughout this work, we assume that the function $\delta(t)$ is non-decreasing, continuous, and such that $\tau(t) \leq \delta(t) \leq t$ for all $t \geq t_{1}$ and some $t_{1} \geq t_{0}$, and $\delta^{n}(t)$ stands for the $n$-fold composition of $\delta(t)$. Let

$$
\psi(t)=\sup _{s \leq t} \tau(s), \quad t \geq t_{0},
$$

and

$$
c(v)=\frac{1-v-\sqrt{1-2 v-v^{2}}}{2}, \quad 0 \leq v \leq \frac{1}{\mathrm{e}} .
$$

(c) The Author(s) 2021. This article is licensed under a Creative Commons Attribution 4.0 International License, which permits use, sharing, adaptation, distribution and reproduction in any medium or format, as long as you give appropriate credit to the original author(s) and the source, provide a link to the Creative Commons licence, and indicate if changes were made. The images or other third party material in this article are included in the article's Creative Commons licence, unless indicated otherwise in a credit line to the material. If material is not included in the article's Creative Commons licence and your intended use is not permitted by statutory regulation or exceeds the permitted use, you will need to obtain permission directly from the copyright holder. To view a copy of this licence, visit http://creativecommons.org/licenses/by/4.0/. 
Also, the notation $\lambda(\xi)$ refers to the smaller real root of the transcendental equation $\lambda=\mathrm{e}^{\lambda \xi}$. Finally, let

$$
k^{*}=\liminf _{t \rightarrow \infty} \int_{\delta(t)}^{t} p(s) d s
$$

The work of Myshkis [27] can be considered as the first systematic study for the oscillation character of the class of the delay differential equations. Recently, these equations have attracted the interest of several researchers, see [1-31]. A huge number of sufficient conditions for the oscillation of Eq. (1) have been obtained. For example, the criteria

$$
\begin{aligned}
& L:=\limsup _{t \rightarrow \infty} \int_{\tau(t)}^{t} p(u) d u>1, \quad \text { where } \tau(t) \text { is non-decreasing, } \\
& k:=\liminf _{t \rightarrow \infty} \int_{\tau(t)}^{t} p(u) d u>\frac{1}{e}
\end{aligned}
$$

were derived respectively in [26] and [23]. In fact, the threshold $\frac{1}{e}$ is of great importance for the oscillation problem of Eq. (1). Since, according to [23], if

$$
\int_{\tau(t)}^{t} p(u) d u \leq \frac{1}{e}
$$

then there exists a non-oscillatory solution of Eq. (1). Indeed, the oscillation problem of Eq. (1) is completely solved when the coefficient and the delay functions are constants $p$ and $\tau$, respectively. In this case all solutions are oscillatory if and only if $p \tau>\frac{1}{e}$; but in the non-autonomous case, the situation is totally different. There is a gap between $\frac{1}{e}$ and

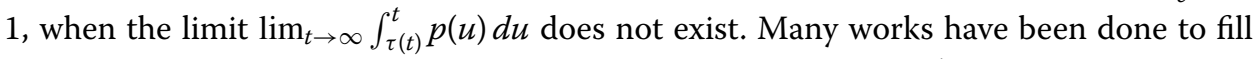
this gap in the case of nondecreasing delays, $L \leq 1$ and $0 \leq k \leq \frac{1}{e}$, see [22, 28] and the references therein. The assumption that the delay is nondecreasing plays a major role in these works. Koplatadze and Kvinikadze [24] showed that many oscillatory criteria can be generalized to equations with non-monotone delay, using a nondecreasing function $\psi(t)$ that is defined as in (2). Since then, several mathematicians have developed and introduced many techniques to study the oscillatory behaviour of these equations. In the following, we show some of these results:

Koplatadze and Kvinikadze [24] improved condition (3) and introduced the sufficient condition

$$
\limsup _{t \rightarrow \infty} \int_{\psi(t)}^{t} p(s) \mathrm{e}^{\int_{\psi(s)}^{\psi(t)} p(u) \Phi_{n}(u) d u} d s>1-c(k)
$$

where

$$
\Phi_{1}(t)=0, \quad \Phi_{n}(t)=\mathrm{e}^{\int_{\tau}^{t}(t) p(s) \Phi_{n-1}(s) d s}, \quad n=2,3, \ldots, \text { for } t \geq t_{0} .
$$

Braverman and Karpuz [4] improved (5) with $n=2$, and obtained

$$
\limsup _{t \rightarrow \infty} \int_{\psi(t)}^{t} p(s) \mathrm{e}^{\int_{\tau(s)}^{\psi(t)} p(u) d u} d s>1
$$


Stavroulakis [29] improved the preceding condition and established

$$
\limsup _{t \rightarrow \infty} \int_{\psi(t)}^{t} p(s) \mathrm{e}^{\int_{\tau(s)}^{\psi(t)} p(u) d u} d s>1-\frac{1-k-\sqrt{1-2 k-k^{2}}}{2}
$$

Infante et al. [21] improved (5) with $n=3$, and (6), and proved that Eq. (1) is oscillatory if

$$
\limsup _{t \rightarrow \infty} \int_{\delta(t)}^{t} p(s) \mathrm{e}^{\int_{\tau(s)}^{\delta(t)} p(u) \mathrm{e}^{\int_{\tau(u)}^{u} p(v) d v} d u} d s>1
$$

or

$$
\limsup _{\epsilon \rightarrow 0^{+}}\left(\limsup _{t \rightarrow \infty} \int_{\delta(t)}^{t} p(s) \mathrm{e}^{(\lambda(k)-\epsilon) \int_{\tau(s)}^{\delta(t)} p(u) d u} d s\right)>1
$$

El-Morshedy and Attia [17] showed that Eq. (1) is oscillatory, if for some $n \in \mathbb{N}$,

$$
\limsup _{t \rightarrow \infty}\left(\int_{\delta(t)}^{t} B_{n}(s) d s+c\left(k^{*}\right) \mathrm{e}^{\int_{\delta(t)}^{t} \sum_{i=0}^{n-1} B_{i}(s) d s}\right)>1,
$$

where

$$
B_{0}(t)=p(t), \quad B_{1}(t)=B_{0}(t) \int_{\tau(t)}^{t} B_{0}(s) \mathrm{e}^{\int_{\tau(s)}^{t} B_{0}(u) d u} d s,
$$

and

$$
B_{n}(t)=B_{n-1}(t) \int_{\delta(t)}^{t} B_{n-1}(s) \mathrm{e}^{\int_{\delta(s)}^{t} B_{n-1}(u) d u} d s, \quad n=2,3, \ldots
$$

In a series of papers, Chatzarakis et al. obtained many oscillatory results for Eq. (1), see [5-15]. For example, Chatzarakis [5] improved (5) with $n=3$, and (6), and obtained the oscillatory condition

$$
\limsup _{t \rightarrow \infty} \int_{h(t)}^{t} p(s) \mathrm{e}^{\int_{\tau(s)}^{h(t)} p(u) M_{n}(u) d u} d s>1-c(k)
$$

where

$$
M_{n}(t)=p(t)\left[1+\int_{\tau(t)}^{t} p(s) \mathrm{e}^{\int_{\tau(s)}^{\delta(t)} M_{n-1}(u) d u} d s\right], \quad M_{0}(t)=p(t)
$$

Bereketoglu et al. [3] improved (11), and proved that Eq. (1) oscillates if there exists $n \in \mathbb{N}$ such that

$$
\limsup _{t \rightarrow \infty} \int_{\delta(t)}^{t} p(s) \mathrm{e}^{\int_{\tau(s)}^{\delta(t)} P_{n}(u) d u} d s>\left\{\begin{array}{l}
1 \quad \text { or } \\
1-c\left(k^{*}\right)
\end{array}\right.
$$

where

$$
P_{0}(t)=p(t)
$$




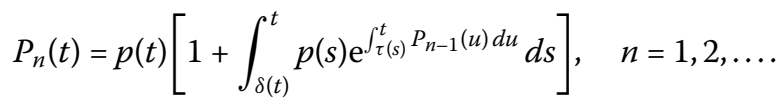

Very recently, Attia, El-Morshedy and Stavroulakis [2] improved (9) and (12), and introduced the following criterion:

$$
\limsup _{t \rightarrow \infty} \int_{\delta(t)}^{t} p(s) \mathrm{e}^{\int_{\tau(s)}^{\delta(t)} p(u) R_{m, n}(u) d u} d s>1-c\left(k^{*}\right),
$$

for some $n, m \in \mathbb{N}$, where

$$
\begin{aligned}
& R_{m, n}(t)=1+\int_{\tau(t)}^{t} p(s) \mathrm{e}^{\int_{\tau(s)}^{t} p(u) D_{m-1, n}(u) d u} d s, \\
& D_{i, j}(t)=\mathrm{e}^{\int_{\tau(t)}^{t} p(s) D_{i, j-1}(s) d s}, \quad i=1,2, \ldots, m-1, j=1,2, \ldots, n,
\end{aligned}
$$

and

$$
\begin{aligned}
& D_{0,0}(t)=\left(\lambda\left(k^{*}\right)-\epsilon\right)\left(1+\left(\lambda\left(k^{*}\right)-\epsilon\right) \int_{\tau(t)}^{\delta(t)} p(s) d s\right), \quad \epsilon \in\left(0, \lambda\left(k^{*}\right)\right), \\
& D_{0, r}(t)=\mathrm{e}^{\int_{\tau(t)}^{t} p(s) D_{0, r-1}(s) d s}, \quad r=1,2, \ldots, n, \\
& D_{i, 0}(t)=R_{i, n}(t), \quad i=1,2, \ldots, m-1 .
\end{aligned}
$$

\section{Results}

Let $x(t)$ be an eventually positive solution of Eq. (1). Then

$$
x^{\prime}(t)+p(t) x(\delta(t)) \leq 0, \quad t \geq t_{1} .
$$

Therefore the following lemmas are applicable to $x(t)$.

Lemma 2.1 ([19, Lemma 2.1.2]) Let $0<k \leq \frac{1}{\mathrm{e}}$. Then

$$
\liminf _{t \rightarrow \infty} \frac{x(\delta(t))}{x(t)} \geq \lambda\left(k^{*}\right)
$$

Lemma $2.2([31])$ Let $k \leq \frac{1}{\mathrm{e}}$. Then

$$
\liminf _{t \rightarrow \infty} \frac{x(t)}{x(\delta(t))} \geq c\left(k^{*}\right)
$$

In the sequel, we define the sequences $\left\{Q_{n}(t)\right\}_{n=0}^{\infty}$ and $\left\{\beta_{n}(t)\right\}_{n=1}^{\infty}$ as follows:

$$
\begin{aligned}
& Q_{0}(t)= \begin{cases}1, & k^{*}=0, \\
\lambda\left(k^{*}\right)-\epsilon, & k^{*}>0, \epsilon \in\left(0, \lambda\left(k^{*}\right)\right),\end{cases} \\
& \beta_{1}(t)=\int_{\delta(t)}^{t} p\left(s_{1}\right) \mathrm{e}^{\int_{\tau\left(s_{1}\right)}^{\delta(t)} p(v) Q_{0}(v) d v} d s_{1}, \quad Q_{1}(t)=\frac{1}{1-\beta_{1}(t)}, \\
& \beta_{2}(t)=\int_{\delta(t)}^{t} p\left(s_{1}\right) d s_{1}+Q_{1}(\delta(t)) \int_{\delta(t)}^{t} p\left(s_{1}\right) \int_{\tau\left(s_{1}\right)}^{\delta(t)} p\left(s_{2}\right) \mathrm{e}^{\int_{\tau\left(s_{2}\right)}^{\delta^{2}(t)} p(v) Q_{1}(v) d v} d s_{2} d s_{1},
\end{aligned}
$$




$$
Q_{2}(t)=\frac{1}{1-\beta_{2}(t)},
$$

and

$$
\begin{aligned}
\beta_{n}(t)= & \int_{\delta(t)}^{t} p\left(s_{1}\right) d s_{1}+Q_{n-1}(\delta(t)) \int_{\delta(t)}^{t} p\left(s_{1}\right) \int_{\tau\left(s_{1}\right)}^{\delta(t)} p\left(s_{2}\right) d s_{2} d s_{1}+\cdots \\
& +\prod_{i=2}^{n-1}\left(Q_{n-1}\left(\delta^{i-1}(t)\right)\right) \int_{\delta(t)}^{t} p\left(s_{1}\right) \int_{\tau\left(s_{1}\right)}^{\delta(t)} p\left(s_{2}\right) \ldots \int_{\tau\left(s_{n-2}\right)}^{\delta^{n-2}(t)} p\left(s_{n-1}\right) d s_{n-1} \cdots d s_{1} \\
& +\prod_{i=2}^{n}\left(Q_{n-1}\left(\delta^{i-1}(t)\right)\right) \\
& \times \int_{\delta(t)}^{t} p\left(s_{1}\right) \int_{\tau\left(s_{1}\right)}^{\delta(t)} p\left(s_{2}\right) \ldots \int_{\tau\left(s_{n-1}\right)}^{\delta^{n-1}(t)} p\left(s_{n}\right) \mathrm{e}^{\int_{\tau}^{\delta^{n}\left(s_{n}\right)} p(v) Q_{n-1}(v) d v} d s_{n} \cdots d s_{1}, \\
Q_{n}(t)= & \frac{1}{1-\beta_{n}(t)}, \quad n=3,4, \ldots
\end{aligned}
$$

The following lemma is essential in order to obtain the main results.

Lemma 2.3 Let $n \in\{0,1,2, \ldots\}$ and $k \leq \frac{1}{\mathrm{e}}$. Then $\beta_{n}<1$, and

$$
\frac{x(\tau(t))}{x(t)} \geq Q_{n}(t) \text { for all sufficiently large } t
$$

where $x(t)$ is a positive solution of Eq. (1).

Proof Since $x(t)$ is a positive solution of Eq. (1), then $x(t)$ is eventually non-increasing for all sufficiently large $t$. Therefore

$$
x^{\prime}(t)+p(t) x(\delta(t)) \leq 0 .
$$

If $k^{*}>0$, then Lemma 2.1 implies, for sufficiently small $\epsilon>0$, that

$$
\frac{x(\tau(t))}{x(t)} \geq \frac{x(\delta(t))}{x(t)} \geq \lambda\left(k^{*}\right)-\epsilon .
$$

This inequality and the non-increasing nature of $x(t)$ lead to

$$
\frac{x(\delta(t))}{x(t)} \geq Q_{0}(t)
$$

On the other hand, dividing Eq. (1) by $x(t)$, integrating from $s$ to $t, s \leq t$, we get

$$
x(s)=x(t) \mathrm{e}^{\int_{s}^{t} p(u) \frac{x(\tau(u))}{x(u)} d u} .
$$

Integrating Eq. (1) from $\delta(t)$ to $t$, we obtain

$$
x(t)-x(\delta(t))+\int_{\delta(t)}^{t} p\left(s_{1}\right) x\left(\tau\left(s_{1}\right)\right) d s_{1}=0 .
$$


Since $\tau\left(s_{1}\right) \leq \delta(t)$ for $s_{1} \leq t,(18)$ and (19) give

$$
x(t)-x(\delta(t))+x(\delta(t)) \int_{\delta(t)}^{t} p\left(s_{1}\right) \mathrm{e}^{\int_{\tau\left(s_{1}\right)}^{\delta(t)} p(u) \frac{x(\tau(u))}{x(u)} d u} d s_{1}=0 .
$$

This equation and (17) lead to

$$
x(t)-x(\delta(t))+x(\delta(t)) \int_{\delta(t)}^{t} p\left(s_{1}\right) \mathrm{e}^{\int_{\tau\left(s_{1}\right)}^{\delta(t)} p(u) Q_{0}(u) d u} d s_{1} \leq 0 .
$$

Consequently,

$$
\frac{x(\tau(t))}{x(t)} \geq \frac{x(\delta(t))}{x(t)} \geq \frac{1}{1-\beta_{1}(t)}=Q_{1}(t) .
$$

Again, integrating Eq. (1) form $\tau\left(s_{1}\right)$ to $\delta(t), s_{1} \leq t$, we obtain

$$
x\left(\tau\left(s_{1}\right)\right)=x(\delta(t))+\int_{\tau\left(s_{1}\right)}^{\delta(t)} p\left(s_{2}\right) x\left(\tau\left(s_{2}\right)\right) d s_{2} .
$$

Substituting into (19), we have

$$
x(t)-x(\delta(t))+x(\delta(t)) \int_{\delta(t)}^{t} p\left(s_{1}\right) d s_{1}+\int_{\delta(t)}^{t} p\left(s_{1}\right) \int_{\tau\left(s_{1}\right)}^{\delta(t)} p\left(s_{2}\right) x\left(\tau\left(s_{2}\right)\right) d s_{2} d s_{1}=0
$$

From this and (18), we obtain

$$
\begin{aligned}
& x(t)-x(\delta(t))+x(\delta(t)) \int_{\delta(t)}^{t} p\left(s_{1}\right) d s_{1} \\
& \quad+x\left(\delta^{2}(t)\right) \int_{\delta(t)}^{t} p\left(s_{1}\right) \int_{\tau\left(s_{1}\right)}^{\delta(t)} p\left(s_{2}\right) \mathrm{e}^{\int_{\tau\left(s_{2}\right)}^{\delta^{2}(t)} p(v) \frac{x(\tau(v))}{x(v)} d v} d s_{2} d s_{1}=0 .
\end{aligned}
$$

Therefore, it follows from (21) that

$$
\begin{aligned}
& x(t)-x(\delta(t))+x(\delta(t)) \int_{\delta(t)}^{t} p\left(s_{1}\right) d s_{1} \\
& \quad+x(\delta(t)) Q_{1}(\delta(t)) \int_{\delta(t)}^{t} p\left(s_{1}\right) \int_{\tau\left(s_{1}\right)}^{\delta(t)} p\left(s_{2}\right) \mathrm{e}^{\int_{\tau\left(s_{2}\right)}^{\delta^{2}(t)} p(v) Q_{1}(v) d v} d s_{2} d s_{1} \leq 0 .
\end{aligned}
$$

Therefore

$$
\frac{x(\delta(t))}{x(t)} \geq \frac{1}{1-\beta_{2}(t)}=Q_{2}(t) .
$$

By simple induction, we get

$$
\begin{aligned}
x(\delta(t))= & x(t)+x(\delta(t)) \int_{\delta(t)}^{t} p\left(s_{1}\right) d s_{1}+x\left(\delta^{2}(t)\right) \int_{\delta(t)}^{t} p\left(s_{1}\right) \int_{\tau\left(s_{1}\right)}^{\delta(t)} p\left(s_{2}\right) d s_{2} d s_{1}+\cdots \\
& +x\left(\delta^{n-1}(t)\right) \int_{\delta(t)}^{t} p\left(s_{1}\right) \int_{\tau\left(s_{1}\right)}^{\delta(t)} p\left(s_{2}\right) \ldots \int_{\tau\left(s_{n-2}\right)}^{\delta^{n-2}(t)} p\left(s_{n-1}\right) d s_{n-1} \cdots d s_{1}
\end{aligned}
$$




$$
+x\left(\delta^{n}(t)\right) \int_{\delta(t)}^{t} p\left(s_{1}\right) \int_{\tau\left(s_{1}\right)}^{\delta(t)} p\left(s_{2}\right) \ldots \int_{\tau\left(s_{n-1}\right)}^{\delta^{n-1}(t)} p\left(s_{n}\right) \mathrm{e}^{\int_{\tau\left(s_{n}\right)}^{\delta^{n}(t)} p(v) \frac{x(\tau(v))}{x(v)} d v} d s_{n} \cdots d s_{1}=0
$$

for $n=3,4, \ldots$ Since

$$
x\left(\delta^{i}(t)\right)=\frac{x\left(\delta^{i}(t)\right)}{x\left(\delta^{i-1}(t)\right)} \cdots \frac{x\left(\delta^{2}(t)\right)}{x(\delta(t))} x(\delta(t)), \quad i=3,4, \ldots, n
$$

we get

$$
x\left(\delta^{i}(t)\right)=x(\delta(t)) \prod_{j=2}^{i} Q_{n-1}\left(\delta^{j-1}(t)\right), \quad i=3,4, \ldots, n .
$$

Substituting into (22), we obtain

$$
\frac{x(\delta(t))}{x(t)} \geq \frac{1}{1-\beta_{n}(t)}=Q_{n}(t), \quad n=3,4, \ldots
$$

Theorem 2.1 Let $n \in\{0,1,2, \ldots\}$. If $\beta_{i} \geq 1, i=1,2, \ldots, n$, or

$$
\limsup _{t \rightarrow \infty} \int_{\delta(t)}^{t} p(s) \mathrm{e}^{\int_{\tau}^{\delta(t)} p(u) Q_{n}(u) d u} d s>1-c\left(k^{*}\right)
$$

then Eq. (1) is oscillatory.

Proof Assume that Eq. (1) has a non-oscillatory solution $x(t)$. Without loss of generality, let $x(t)$ be an eventually positive solution. By using (20), we obtain

$$
x(t)-x(\delta(t))+x(\delta(t)) \int_{\delta(t)}^{t} p\left(s_{1}\right) \mathrm{e}^{\int_{\tau\left(s_{1}\right)}^{\delta(t)} p(u) \frac{x(\tau(u))}{x(u)} d u} d s_{1}=0
$$

Using Lemma 2.2, it follows that

$$
\int_{\delta(t)}^{t} p\left(s_{1}\right) \mathrm{e}^{\int_{\tau\left(s_{1}\right)}^{\delta(t)} p(u) Q_{n}(u) d u} d s_{1} \leq 1-\frac{x(t)}{x(\delta(t))}
$$

From this and Lemma 2.2, we obtain

$$
\limsup _{t \rightarrow \infty} \int_{\delta(t)}^{t} p\left(s_{1}\right) \mathrm{e}^{\int_{\tau\left(s_{1}\right)}^{\delta(t)} p(u) Q_{n}(u) d u} d s_{1} \leq 1-c\left(k^{*}\right)
$$

This contradicts (23).

Theorem 2.2 Assume that $n \in\{0,1,2, \ldots\}$ and $\delta(t)$ is a strictly increasing for $t \geq t_{1}$. If $\beta_{i} \geq 1, i=1,2, \ldots, n$, or

$$
\limsup _{t \rightarrow \infty}\left(\frac{\int_{t}^{\delta^{-1}(t)} p\left(s_{1}\right) \int_{\tau\left(s_{1}\right)}^{t} p\left(s_{2}\right) d s_{2} d s_{1}}{1-\int_{t}^{\delta^{-1}(t)} p\left(s_{1}\right) d s_{1}}+\int_{\delta(t)}^{t} p\left(s_{1}\right) \mathrm{e}^{\int_{\tau\left(s_{1}\right)}^{\delta(t)} p(u) Q_{n}(u) d u} d s_{1}\right)>1
$$

then Eq. (1) is oscillatory. 
Proof Assume that there exists a positive solution $x(t)$ of Eq. (1). From the proof of Theorem 2.1 , we see that

$$
\frac{x(t)}{x(\delta(t))}+\int_{\delta(t)}^{t} p\left(s_{1}\right) \mathrm{e}^{\int_{\tau\left(s_{1}\right)}^{\delta(t)} p(u) Q_{n}(u) d u} d s_{1} \leq 1
$$

Integrating Eq. (1) from $t$ to $\delta^{-1}(t)$, we have

$$
x\left(\delta^{-1}(t)\right)-x(t)+\int_{t}^{\delta^{-1}(t)} p\left(s_{1}\right) x\left(\tau\left(s_{1}\right)\right) d s_{1}=0 .
$$

Since $t \geq \tau\left(s_{1}\right)$ for $\delta^{-1}(t) \geq s_{1}$, one has

$$
x\left(\tau\left(s_{1}\right)\right)=x(t)+\int_{\tau\left(s_{1}\right)}^{t} p\left(s_{2}\right) x\left(\tau\left(s_{2}\right)\right) d s_{2} .
$$

From this, (26) and the non-increasing nature of $x(t)$, we have

$$
x\left(\delta^{-1}(t)\right)-x(t)+x(t) \int_{t}^{\delta^{-1}(t)} p\left(s_{1}\right) d s_{1}+x(\delta(t)) \int_{t}^{\delta^{-1}(t)} p\left(s_{1}\right) \int_{\tau\left(s_{1}\right)}^{t} p\left(s_{2}\right) d s_{2} d s_{1} \leq 0,
$$

which in turn leads to

$$
\frac{x(t)}{x(\delta(t)} \geq \frac{\int_{t}^{\delta^{-1}(t)} p\left(s_{1}\right) \int_{\tau\left(s_{1}\right)}^{t} p\left(s_{2}\right) d s_{2} d s_{1}}{1-\int_{t}^{\delta^{-1}(t)} p\left(s_{1}\right) d s_{1}}
$$

By substituting into (25), we have

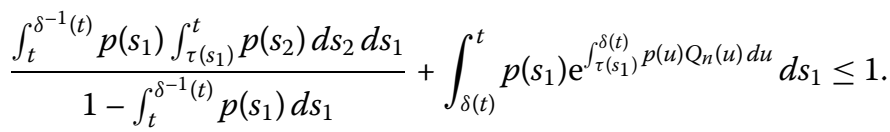

Taking the upper limits of both sides as $t$ goes to $\infty$, we obtain a contradiction with (24).

Theorem 2.3 Let $n \in\{0,1,2, \ldots\}$. If $\beta_{i} \geq 1, i=1,2, \ldots, n$, or

$$
\limsup _{t \rightarrow \infty} \int_{\delta(t)}^{t} \frac{p\left(s_{1}\right) \mathrm{e}^{\int_{\tau}^{\delta\left(s_{1}\right)} p(u) Q_{n}(u) d u}}{1-\int_{\delta\left(s_{1}\right)}^{\delta(t)} p\left(s_{2}\right) \mathrm{e}^{\int_{\tau\left(s_{2}\right)}^{\delta\left(s_{1}\right)} p(u) Q_{n}(u) d u} d s_{2}} d s_{1}>1-c\left(k^{*}\right)
$$

then Eq. (1) is oscillatory.

Proof As before, let $x(t)$ be a positive solution of Eq. (1). Then

$$
x(t)-x(\delta(t))+\int_{\delta(t)}^{t} p\left(s_{1}\right) x\left(\tau\left(s_{1}\right)\right) d s_{1}=0 .
$$

By using (17), from the proof of Theorem 2.3, we have

$$
x(t)-x(\delta(t))+\int_{\delta(t)}^{t} p\left(s_{1}\right) x\left(\delta\left(s_{1}\right)\right) \mathrm{e}^{\int_{\tau\left(s_{1}\right)}^{\delta\left(s_{1}\right)} p(u) \frac{x(\tau(u))}{x(u)} d u} d s_{1}=0 .
$$


Integrating Eq. (1) form $\delta\left(s_{1}\right)$ to $\delta(t), s_{1} \leq t$, we get

$$
x(\delta(t))-x\left(\delta\left(s_{1}\right)\right)+\int_{\delta\left(s_{1}\right)}^{\delta(t)} p\left(s_{2}\right) x\left(\tau\left(s_{2}\right) d s_{2}=0 .\right.
$$

Since $\tau\left(s_{2}\right) \leq \delta\left(s_{1}\right)$ for $s_{2} \leq s_{1}$, it follows from (17) and (30) that

$$
x(\delta(t))-x\left(\delta\left(s_{1}\right)\right)+x\left(\delta\left(s_{1}\right)\right) \int_{\delta\left(s_{1}\right)}^{\delta(t)} p\left(s_{2}\right) \mathrm{e}^{\int_{\tau\left(s_{2}\right)}^{\delta\left(s_{1}\right)} p(u) \frac{x(\tau(u))}{x(u)} d u} d s_{2}=0
$$

that is,

$$
x\left(\delta\left(s_{1}\right)\right)=\frac{x(\delta(t))}{1-\int_{\delta\left(s_{1}\right)}^{\delta(t)} p\left(s_{2}\right) \mathrm{e}^{\int_{\tau\left(s_{2}\right)}^{\delta\left(s_{1}\right)} p(u) \frac{x(\tau(u))}{x(u)} d u} d s_{2}} .
$$

From this, (29) and Lemma 2.3, we have

$$
\int_{\delta(t)}^{t} \frac{p\left(s_{1}\right) \mathrm{e}^{\int_{\tau\left(s_{1}\right)}^{\delta\left(s_{1}\right)} p(u) Q_{n}(u) d u}}{1-\int_{\delta\left(s_{1}\right)}^{\delta(t)} p\left(s_{2}\right) \mathrm{e}^{\int_{\tau\left(s_{2}\right)}^{\delta\left(s_{1}\right)} p(u) Q_{n}(u) d u} d s_{2}} d s_{1} \leq 1-\frac{x(t)}{x(\delta(t))} .
$$

This, together with Lemma 2.2, implies that

$$
\limsup _{t \rightarrow \infty} \int_{\delta(t)}^{t} \frac{p\left(s_{1}\right) \mathrm{e}^{\int_{\tau\left(s_{1}\right)}^{\delta\left(s_{1}\right)} p(u) Q_{n}(u) d u}}{1-\int_{\delta\left(s_{1}\right)}^{\delta(t)} p\left(s_{2}\right) \mathrm{e}^{\int_{\tau\left(s_{2}\right)}^{\delta\left(s_{1}\right)} p(u) Q_{n}(u) d u} d s_{2}} d s_{1} \leq 1-c\left(k^{*}\right) .
$$

This is a contradiction.

The proof of the following result is the same as those of Theorems 2.1 and 2.2, and hence it will be omitted.

Theorem 2.4 Let $n \in\{0,1,2, \ldots\}$ and $\delta(t)$ be strictly increasing for $t \geq t_{1}$. If $\beta_{i} \geq 1, i=$ $1,2, \ldots, n$, or

$$
\begin{aligned}
& \limsup _{t \rightarrow \infty}\left(\frac{\int_{t}^{\delta^{-1}(t)} p\left(s_{1}\right) \int_{\tau\left(s_{1}\right)}^{t} p\left(s_{2}\right) d s_{2} d s_{1}}{1-\int_{t}^{\delta^{-1}(t)} p\left(s_{1}\right) d s_{1}}+\int_{\delta(t)}^{t} \frac{p\left(s_{1}\right) \mathrm{e}^{\int_{\tau\left(s_{1}\right)}^{\delta\left(s_{1}\right)} p(u) Q_{n}(u) d u}}{1-\int_{\delta\left(s_{1}\right)}^{\delta(t)} p\left(s_{2}\right) \mathrm{e}^{\int_{\tau\left(s_{2}\right)}^{\delta\left(s_{1}\right)} p(u) Q_{n}(u) d u} d s_{2}} d s_{1}\right) \\
& \quad>1 .
\end{aligned}
$$

then Eq. (1) is oscillatory.

\section{Remark 2.1}

- The criterion (23) improves conditions (5) with $n=2$, (6), (7), and (9) when $k=0$.

- Lemma 2.3 can be used to improve and generalize the oscillation results of [30, Lemma 2.1], [16, Theorem 2.6] and [18, Lemma 2.5].

Example 2.1 Consider the first order delay differential equation

$$
x^{\prime}(t)+p(t) x(\tau(t))=0, \quad t \geq 2,
$$


Attia Advances in Difference Equations

(2021) 2021:41

Page 10 of 12

where

$$
\tau(t)=t-1-\zeta \cos ^{2}\left(\frac{\nu \pi(2 t+1)}{2}\right), \quad v=10,000, \zeta=0.0001,
$$

and

$$
p(t):= \begin{cases}0, & t \in\left[\xi_{i}, \mu_{i}\right], \\ \gamma\left(t-\mu_{i}\right), & t \in\left[\mu_{i}, \mu_{i}+1\right], \\ \gamma, & t \in\left[\mu_{i}+1, \mu_{i}+9\right], \\ \frac{\gamma\left(t-\xi_{i+1}\right)}{\mu_{i}+9-\xi_{i+1}}, & t \in\left[\mu_{i}+9, \xi_{i+1}\right],\end{cases}
$$

where $i, \mu_{i} \in \mathbb{N}, \gamma=0.4195, \xi_{1} \geq 0, \mu_{i}>1+\xi_{i}$, and $\xi_{i+1}>\mu_{i}+9$ such that $\lim _{i \rightarrow \infty} \xi_{i}=\infty$.

Since

$$
0 \leq \liminf _{t \rightarrow \infty} \int_{\tau(t)}^{t} p(s) d s \leq \lim _{i \rightarrow \infty} \int_{\tau\left(\mu_{i}\right)}^{\mu_{i}} p(s) d s=\lim _{i \rightarrow \infty} \int_{\mu_{i}-1}^{\mu_{i}} p(s) d s=0,
$$

one has $k=k^{*}=0$, and it follows that conditions (4), (9) and (13) fail to apply. Let $\delta(t)=t-1$ and

$$
I(t)=\frac{\int_{t}^{\delta^{-1}(t)} p\left(s_{1}\right) \int_{\tau\left(s_{1}\right)}^{t} p\left(s_{2}\right) d s_{2} d s_{1}}{1-\int_{t}^{\delta^{-1}(t)} p\left(s_{1}\right) d s_{1}}+\int_{\delta(t)}^{t} p\left(s_{1}\right) \mathrm{e}^{\int_{\tau\left(s_{1}\right)}^{\delta(t)} p(u) Q_{2}(u) d u} d s_{1} .
$$

Since

$$
I\left(\mu_{i}+8\right) \geq \frac{\int_{\mu_{i}+8}^{\mu_{i}+9} p\left(s_{1}\right) \int_{s_{1}-1}^{\mu_{i}+8} p\left(s_{2}\right) d s_{2} d s_{1}}{1-\int_{\mu_{i}+8}^{\mu_{i}+9} p\left(s_{1}\right) d s_{1}}+\int_{\mu_{i}+7}^{\mu_{i}+8} p\left(s_{1}\right) \mathrm{e}^{\int_{s_{1}-1}^{\mu_{i}+7} p(u) Q_{2}(u) d u} d s_{1} .
$$

Then

$$
Q_{2}(u)=\frac{1}{1-\beta_{2}(u)}, \quad \mu_{i}+6 \leq u \leq \mu_{i}+7
$$

and

$$
\beta_{2}(u) \geq \int_{u-1}^{u} p\left(s_{1}\right) d s_{1}+Q_{1}(u-1) \int_{u-1}^{u} p\left(s_{1}\right) \int_{s_{1}-1}^{u-1} p\left(s_{2}\right) \mathrm{e}^{\int_{s_{2}-1}^{u-2} p(v) Q_{1}(v) d v} d s_{2} d s_{1},
$$

where $\mu_{i}+3 \leq v \leq \mu_{i}+5$, also

$$
Q_{1}(v)=\frac{1}{1-\beta_{1}(v)}
$$

and

$$
\beta_{1}(v) \geq \int_{v-1}^{v} p\left(s_{1}\right) \mathrm{e}^{\int_{s_{1}-1}^{v-1} p(r) Q_{0}(r) d r} d s_{1}, \quad \mu_{i}+1 \leq r \leq \mu_{i}+4 .
$$

Then

$$
\beta_{1}(v) \geq \int_{\nu-1}^{v} \gamma \mathrm{e}^{\gamma\left(v-s_{1}\right)}=\mathrm{e}^{\gamma}-1 \quad \text { and } \quad Q_{1}(v) \geq \frac{1}{2-\mathrm{e}^{\gamma}},
$$


also

$$
Q_{2}(u) \geq \frac{A}{A-\mathrm{e}^{\gamma A}+1}, \quad A=\frac{1}{2-\mathrm{e}^{\gamma}} .
$$

Therefore $I\left(\mu_{i}+8\right)>1.0002$. As a consequence, $\limsup _{t \rightarrow \infty} I(t)>1$, and hence Theorem 2.2 implies that Eq. (32) is oscillatory. However, if we assume that $\delta(t)=\psi(t)$ (which is defined as in (2)), then

$$
t-1-\zeta \leq \tau(t) \leq \delta(t) \leq t-1
$$

Consequently, $\int_{\tau(t)}^{t} p(s) d s \leq(1+\zeta) \gamma$. Then, $\Phi_{8}(t)<3.363136$, and it follows that

$$
\limsup _{t \rightarrow \infty} \int_{\delta(t)}^{t} p(s) \mathrm{e}^{\int_{\tau(s)}^{\delta(t)} p(u) \Phi_{8}(u) d u} d s \leq \limsup _{t \rightarrow \infty} \int_{t-1-\zeta}^{t} \gamma \mathrm{e}^{\int_{s-1-\zeta}^{t-1} \gamma \Phi_{8}(u) d u} d s<1 .
$$

Therefore, none of conditions (5) with $n=8$ or (6)-(8) hold. Also, since

$$
\limsup _{t \rightarrow \infty}\left(\int_{\delta(t)}^{t} B_{2}(s) d s+c\left(k^{*}\right) \mathrm{e}^{\int_{\delta(t)}^{t} \sum_{r=0}^{1} B_{r}(s) d s}\right) \leq \limsup _{t \rightarrow \infty} \int_{t-1-\zeta}^{t} B_{2}(s) d s<0.18315<1,
$$

condition (10) with $n=2$ fails to apply. Finally,

$$
\underset{t \rightarrow \infty}{\limsup } \int_{\delta(t)}^{t} p(s) \mathrm{e}^{\int_{\tau(s)}^{\delta(t)} P_{3}(u) d u} d s<\limsup _{t \rightarrow \infty} \int_{t-1-\zeta}^{t} \gamma \mathrm{e}^{1.2115 \int_{s-1-\zeta}^{t-1} \gamma d u} d s<0.817<1,
$$

hence, condition (12) with $n=3$ is not satisfied.

\section{Conclusion}

In this work, we obtained new oscillatory criteria for Eq. (1), using improved lower bounds for the quantity $\frac{x(\tau(u))}{x(t)}$, where $x(t)$ is any positive solution of Eq. (1). Some of the obtained results improve many previous works. Finally, we introduced an example to demonstrate the simplicity and efficiency of some of our results, especially when $k=0$.

\section{Acknowledgements}

The author would like to express his deep thanks to Prof. Hassan. A. El-Morshedy for his help and suggestions. Also the author would like to express his gratitude to the anonymous referees for their help in improving the manuscript.

Funding

This project was supported by the Deanship of Scientific Research at Prince Sattam Bin Abdulaziz University under the research project 2020/01/16630.

Abbreviations

$\delta^{n}(t)$ stands for the $n$-fold composition of $\delta(t) ; \mathbb{N}$ denotes the set of natural numbers.

Availability of data and materials

No datasets were generated during this work.

Competing interests

The author declares that they have no competing interests. 


\section{Publisher's Note}

Springer Nature remains neutral with regard to jurisdictional claims in published maps and institutional affiliations.

\section{Received: 1 December 2020 Accepted: 29 December 2020 Published online: 09 January 2021}

\section{References}

1. Agarwal, R.P., Berezansky, L., Braverman, E., Domoshnitsky, A.: Non-Oscillation Theory of Functional Differential Equations with Applications. Springer, New York (2012)

2. Attia, E.R., El-Morshedy, H.A., Stavroulakis, I.P.: Oscillation criteria for first order differential equations with non-monotone delays. Symmetry 12, 718 (2020)

3. Bereketoglu, H., Karakoc, F., Oztepe, G.S., Stavroulakis, I.P.: Oscillation of first order differential equations with several non-monotone retarded arguments. Georgian Math. J. 27, 341-350 (2020)

4. Braverman, E., Karpuz, B.: On oscillation of differential and difference equations with non-monotone delays. Appl. Math. Comput. 218, 3880-3887 (2011)

5. Chatzarakis, G.E.: On oscillation of differential equations with non-monotone deviating arguments. Mediterr. J. Math. $14,17(2017)$

6. Chatzarakis, G.E.: An oscillation criterion in delay differential equations. Gen. Lett. Math. 5, 1-6 (2018)

7. Chatzarakis, G.E.: Oscillations of equations caused by several deviating arguments. Opusc. Math. 39, 321-353 (2019)

8. Chatzarakis, G.E.: Oscillation test for linear deviating differential equations. Appl. Math. Lett. 98, 352-358 (2019)

9. Chatzarakis, G.E.: Oscillation of deviating differential equations. Math. Bohem. 145, 435-448 (2020)

10. Chatzarakis, G.E., Jadlovská, I.: Improved iterative oscillation tests for first-order deviating differential equations. Opusc. Math. 38, 327-356 (2018)

11. Chatzarakis, G.E., Jadlovská, I.: Oscillations in deviating differential equations using an iterative method. Commun. Math. 27, 143-169 (2019)

12. Chatzarakis, G.E., Jadlovská, I.: Oscillations in differential equations caused by non-monotone arguments. Nonlinear Stud. 27, 589-607 (2020)

13. Chatzarakis, G.E., Jadlovská, I., Li, T.: Oscillations of differential equations with non-monotone deviating arguments. Adv. Differ. Equ. 2019, 20 (2019)

14. Chatzarakis, G.E., Li, T.: Oscillations of differential equations generated by several deviating arguments. Adv. Differ. Equ. 2017, 24 (2017)

15. Chatzarakis, G.E., Li, T.: Oscillation criteria for delay and advanced differential equations with non-monotone arguments. Complexity 2018, 18 (2018)

16. El-Morshedy, H.A.: On the distribution of zeros of solutions of first order delay differential equations. Nonlinear Anal. 74, 3353-3362 (2011)

17. El-Morshedy, H.A., Attia, E.R.: New oscillation criterion for delay differential equations with non-monotone arguments. Appl. Math. Lett. 54, 54-59 (2016)

18. El-Morshedy, H.A., Attia, E.R.: On the distance between adjacent zeros of solutions of first order differential equations with distributed delays. Electron. J. Qual. Theory Differ. Equ. 2016, 8 (2016)

19. Erbe, L.H., Zhang, B.G.: Oscillation for first order linear differential equations with deviating arguments. Differ. Integral Equ. 1, 305-314 (1988)

20. Gyori, I., Ladas, G.: Oscillation Theory of Delay Differential Equations with Applications. Clarendon Press, Oxford (1991)

21. Infante, G., Koplatadze, R., Stavroulakis, I.P.: Oscillation criteria for differential equations with several retarded arguments. Funkc. Ekvacioj 58, 347-364 (2015)

22. Kon, M., Sficas, Y.G., Stavroulakis, I.P.: Oscillation criteria for delay equations. Proc. Am. Math. Soc. 128, 2989-2997 (2000)

23. Koplatadze, R.G., Chanturija, T.A.: On oscillatory and monotonic solutions of first order differential equations with deviating arguments. Differ. Uravn. 18, 1463-1465 (1982) (in Russian)

24. Koplatadze, R.G., Kvinikadze, G.: On the oscillation of solutions of first order delay differential inequalities and equations. Georgian Math. J. 1, 675-685 (1994)

25. Ladas, G.: Sharp conditions for oscillations caused by delays. Appl. Anal. 9, $93-98$ (1979)

26. Ladas, G., Lakshmikantham, V., Papadakis, L.S.: Oscillations of higher-order retarded differential equations generated by the retarded arguments. In: Delay and Functional Differential Equations and Their Applications. Academic Press, New York (1972)

27. Myshkis, A.D.: Linear homogeneous differential equations of first order with deviating arguments. Usp. Mat. Nauk 5, 160-162 (1950) (Russian)

28. Sficas, Y.G., Stavroulakis, I.P.: Oscillation criteria for first-order delay equations. Bull. Lond. Math. Soc. 35, 239-246 (2003)

29. Stavroulakis, I.P.: Oscillation criteria for delay and difference equations with non-monotone arguments. Appl. Math. Comput. 226, 661-672 (2014)

30. Wu, H.W., Erbe, L., Peterson, A.C.: Upper bounds for the distances between consecutive zeros of solutions of first order delay differential equations. J. Math. Anal. Appl. 229, 562-575 (2015)

31. Yu, J.S., Wang, Z.C., Zhang, B.G., Qian, X.Z.: Oscillations of differential equations with deviating arguments. Panam. Math. J. 2, 59-78 (1992) 